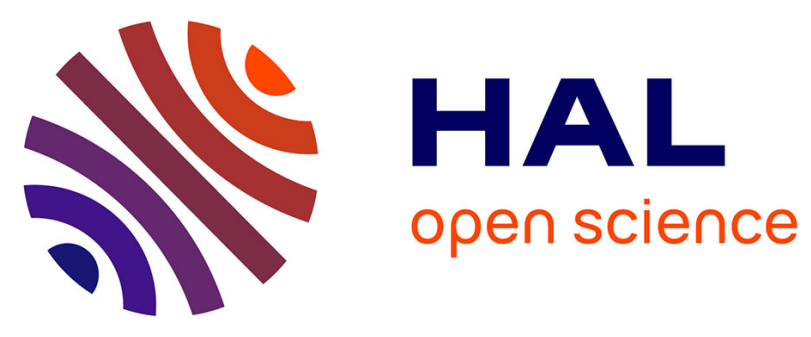

\title{
Lyapunov Stability Margins for humanoid robot balancing
}

Emmanouil Spyrakos-Papastavridis, Nicolas Perrin, Nikos G Tsagarakis, Jian S. Dai, Darwin G. Caldwell

\section{- To cite this version:}

Emmanouil Spyrakos-Papastavridis, Nicolas Perrin, Nikos G Tsagarakis, Jian S. Dai, Darwin G. Caldwell. Lyapunov Stability Margins for humanoid robot balancing. 2014 IEEE/RSJ International Conference on Intelligent Robots and Systems, Sep 2014, Chicago, IL, United States. 10.1109/IROS.2014.6942673 . hal-01394903

\section{HAL Id: hal-01394903 https://hal.sorbonne-universite.fr/hal-01394903}

Submitted on 14 Nov 2016

HAL is a multi-disciplinary open access archive for the deposit and dissemination of scientific research documents, whether they are published or not. The documents may come from teaching and research institutions in France or abroad, or from public or private research centers.
L'archive ouverte pluridisciplinaire $\mathbf{H A L}$, est destinée au dépôt et à la diffusion de documents scientifiques de niveau recherche, publiés ou non, émanant des établissements d'enseignement et de recherche français ou étrangers, des laboratoires publics ou privés. 


\title{
Lyapunov Stability Margins for Humanoid Robot Balancing
}

\author{
Emmanouil Spyrakos-Papastavridis, Nicolas Perrin, Nikos G. Tsagarakis, \\ Jian S. Dai and Darwin G. Caldwell
}

\begin{abstract}
This work introduces a novel balance monitoring strategy for humanoid robots. The proposed method addresses the problem of ensuring the balance maintenance of a humanoid robot, through the online monitoring of its state of balance by means of a Lyapunov (energy) function. The proposed method involves the use of dynamical models accounting for both the link and motor states. Energy limits corresponding to the front and rear edges of the support polygon are computed using a closed-loop Lyapunov function. Therefore, this method focuses on the resolution of two issues through a single control scheme, namely, guaranteeing asymptotical stability of the robot at the joint level, in addition to ensuring that it maintains its dynamical balance. A mathematical proof of the previous claims, as well as of the method's validity, is provided in the paper, whereby a direct relationship between the CoP and the system's energy has been established for the first time. Experimental results of step recovery and walking tests performed on the COmpliant huMANoid (COMAN) corroborate the method's applicability and performance as a balance monitor.
\end{abstract}

\section{INTRODUCTION}

$\mathrm{T}$ HE topic of humanoid balancing has been studied extensively as its importance has undergone a steady increase in recent years, owing to the development of numerous humanoids possessing distinct mechanical structures. Among the most important contributions that aided in the shaping of the theories developed thereafter, was the introduction of the zero-moment-point (ZMP), that has been offered to the field through the work presented in [1], wherein the authors propose a point under the foot sole whose position defines the dynamic balance of a bipedal system. There have also been noteworthy extensions of this theory that have aided in the clarification of certain aspects pertaining to the original ZMP notion. The work in [2] rigorously describes the center of pressure $(\mathrm{CoP})$ and $\mathrm{ZMP}$ concepts, highlighting their differences as well as the cases in which they are equivalent. Another important balance criterion known as the foot rotation indicator (FRI) [3] has been defined as the point on a humanoid's foot at which the net normal force must act in order to ensure that the foot remains stationary, although this only applies to the single

E. Spyrakos-Papastavridis, N. G. Tsagarakis and D. G. Caldwell are with the Department of Advanced Robotics, Istituto Italiano di Tecnologia, via Morego, 30, 16163 Genova, Italy \{emmanouil.spyrakos, nikos.tsagarakis, darwin.caldwell $\}$ at iit.it

Nicolas Perrin is with Institut des Systèmes Intelligents et de Robotique, CNRS UMR 7222 \& Université Pierre et Marie Curie, 4 place Jussieu, 75252 Paris cedex 05, France \{perrin@isir.upmc.fr

Jian S. Dai is with the Centre for Robotics Research, King's College London, Strand, London, WC2R 2LS, England, United Kingdom \{jian.dai\} at kcl.ac.uk support stance. As far as balancing strategies are concerned, there have been several works dealing with this topic. [4] proposes dynamic balancing through the use of ankle and hip strategies. The former involves movement of the entire system about the ankle and has been studied in [5] and [6], where the authors have utilized torque control of the ankles. [6] assumes a direct torque control of the stiff ankle, rendering it possible to control the $\mathrm{CoP}$ in a delay-free manner, whereas this paper demonstrates how the presence of under-actuated elastic elements in compliant humanoids prevents the latter from holding true. A natural extension of these schemes, namely the hip strategy, has been examined in addition to how a humanoid can switch between various stabilization techniques [6], i.e. hip/ankle strategies, and stepping, to ensure that stability is maintained. [7] proposes a method of passivating a humanoid through a combination of translating the ground reaction forces (GRFs) to joint torques and gravity compensation control. [8] also focuses on a passivity-based approach that is aimed at absorbing the GRFs acting on a humanoid during walking. [9] demonstrates that the monitoring and modification of the rate of change of angular momentum as both a stability criterion and control variable, can be used to balance a bipedal robot. ZMP preview control schemes are a common choice for stabilization and have been described in [10][11][12], while ZMP feedback controllers have been presented and adapted to the field of stepping in the presence of external perturbations [13]. This leads to the next topic of stepping, where [14] provides an answer to the question of 'when and where must a step be taken' in order to stabilize a given system, by introducing the Capture Region concept. These are regions on the terrain, within which a robot can take a step in order to stabilize its structure. The problem of humanoid balance monitoring and fall detection has been approached using several methods, ranging from the utilization of attitude sensors and reflexes [15], to viability theory [16] and probabilistic techniques [17], while it is crucial to mention the utilization of the Maximal Output Admissible (MOA) set technique that was recently proposed [18]. Contrarily, this paper aims at addressing the aforementioned problem through the consideration of dynamical models of increased complexity and closed-loop energy functions that are used to generate values corresponding to unstable states, from which the robot would not be capable of recovering its balance. The idea of utilizing Lyapunov Stability Margins for the assessment of a robot's dynamical balance, was initially introduced in the pioneering work seen in [19], for two reasons. Firstly, the ZMP/CoP is incapable of providing a useful measure of stability on uneven terrain and secondly, the Energy Stability Margins unanimously conclude that tipping over a contact point edge is an unstable 
situation for walking systems, even though this phenomenon is frequently encountered in stable walking [19]. While [19] introduced the Lyapunov Stability Margin concept without giving a practical and computationally tractable example, our proposed approach contributes by providing a specific Lyapunov Stability Margin for a humanoid robot that can be computed quickly through a simple relationship between the CoP deviation and the closed-loop energy using specific Lyapunov functions. Furthermore, it distinguishes itself from the orbital energy [20] and energy stability margin methods [21] of monitoring balance, in the sense that it considers not only more intricate dynamical models that include compliance but it also accounts for the closed-loop system properties. An additional contribution of this work is that it presents a control scheme that ensures the closed-loop system's asymptotical stability and offers a method, based on this same control scheme, through which the robot's dynamical stability can be monitored.

The rest of the paper is structured as follows; section II introduces the dynamical models and the joint controller, section III describes the balance controllability, section IV provides a mathematical proof of the method's validity, section $\mathrm{V}$ reports on the experimental results, section VI extends the relevant theory to walking, and finally section VII discusses the conclusions.

\section{DYNAMICAL MODEL AND JoINT CONTROL}

\section{A. Compliant Robot Dynamics}

For the double support case, we consider a generic $j$-degree of freedom robot with $n_{i}$-drives for each degree of freedom $i=1,2 \ldots j$. The total number of drives is $n=\sum_{i=1}^{j} n_{i}$. The link and motor dynamics may be described as follows [22]:

$$
\begin{gathered}
M_{J}(q) \ddot{q}+N \dot{q}+C(q, \dot{q}) \dot{q}+S_{m}^{T} P\left(S_{m} q-\theta\right)=\tau_{g}(q) \\
J \ddot{\theta}+D \dot{\theta}-P S_{m} q+P \theta=V_{T G} V_{m}
\end{gathered}
$$

where $q$ and $\theta$ are the link and motor positions, $M_{J}(q), N$ and $C(q, \dot{q}) \in R^{j \times j}$ are the inertia, damping and Coriolis/centripetal matrices respectively, $\tau_{g}(q)$ is the gravity torque vector, $V_{T G} \in R^{n \times n}$ is the voltage-to-torque gain matrix and $V_{m}$ represents the motor voltages. $P \in R^{n \times n}$ is a diagonal matrix with positive entries representing the passive spring stiffness between the motors and the robot links, while $J, D \in R^{n \times n}$ are the motor inertia and damping. $S_{m}^{T} \in R^{j \times n}$ possesses the following structure:

$$
S_{m}^{T}=\left[\begin{array}{cccc}
\underbrace{11 \cdots 1}_{n_{1}} & 0 & \cdots & 0 \\
0 & \underbrace{11 \cdots 1}_{n_{2}} & \cdots & 0 \\
\vdots & \vdots & \ddots & \vdots \\
0 & 0 & \cdots & \underbrace{11 \cdots 1}_{n_{j}}
\end{array}\right]
$$

A substantial degree of modeling accuracy was preserved by including all the sagittal joints in the system's mathematical description. However, the over-actuated nature of the double support stance led to the six joints being treated as three (Fig. 1), although all six actuators were considered. This approach provides greater precision as compared to approximating the whole humanoid's structure as a single inverted pendulum. Fig. 1 depicts the correspondence between the actual robot's joints with those of the model. The elements of the 3-DOF double support vector were arranged as follows:

$$
\tau_{g 0}=\left[\begin{array}{lll}
\tau_{g a} & \tau_{g k} & \tau_{g h}
\end{array}\right]^{T}
$$

with $\tau_{a}, \tau_{k}, \tau_{h}$ representing the ankle, knee and hip torques respectively. One of the main properties of the gravity vector, to be used in subsequent sections, is the following:

$$
\left\|\frac{\partial \tau_{g}(q)}{\partial q}\right\|=\left\|\frac{\partial^{2} U_{g}(q)}{\partial q^{2}}\right\|<\alpha
$$

for some $\alpha>0$; here $U_{g}(q)$ denotes the potential energy due to gravity, $\tau_{g}(q)=-\left(\partial U_{g}(q) / \partial q\right)^{T}$ and the operator norm $\|A\|=\max \{\|A x\| /\|x\|\}$ is considered.
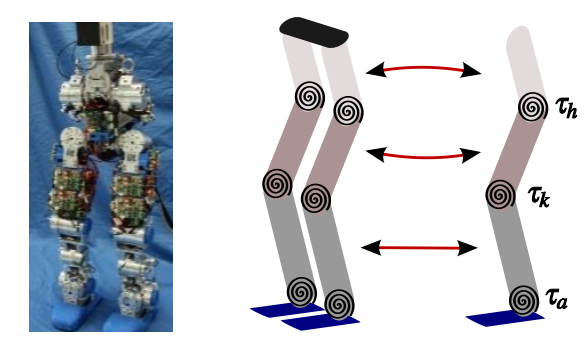

Figure 1. Lower body of compliant humanoid COMAN and corresponding double support model.

\section{B. Desired Gravity Compensation Controller}

The low-level joint controller was based upon gravity compensation control, employing both motor and link feedback. The associated control law may be represented as follows:

$$
V_{m}=K_{m 1}\left(S_{m} q_{d}-\theta\right)-K_{m 2} \dot{\theta}-K_{j 2} \dot{q}+u_{g c}
$$

where $u_{g c}$ is the gravity compensation term and $q_{d}$ is the desired position, while $K_{m 1}, K_{m 2}$ and $K_{j 2}$ are the diagonal, positive definite motor position, motor velocity and link velocity feedback gain matrices.

For the double support, $u_{g c}$ is given by:

$$
u_{g c}=-V_{T G}^{-1}\left(V_{T G} K_{m 1} P^{-1}+I\right)\left(S_{m}^{T}\right)^{+} \tau_{g}\left(q_{d}\right)
$$

where $q$ has been replaced with $q_{d}$ since we are considering a desired gravity compensation controller, while $\left(S_{m}^{T}\right)^{+}$ denotes the Moore-Penrose pseudoinverse of $S_{m}^{T}$. By considering the closed-loop system's steady state equations, we can define the following matrix that plays a central role in the stability analysis since it must satisfy certain 
conditions in order for closed-loop stability to be guaranteed, as shown in [23]:

$$
T_{D}=\left[\begin{array}{cc}
S_{m}^{T} P S_{m} & -S_{m}^{T} P \\
-P S_{m} & V_{T G} K_{m 1}+P
\end{array}\right]
$$

The desired motor and link positions are related by:

$$
\theta_{d}=S_{m} q_{d}-P^{-1}\left(S_{m}^{T}\right)^{+} \tau_{g}\left(q_{d}\right)
$$

Additionally, the following matrix is defined:

$$
\gamma=N \cdot\left(D+V_{T G} K_{m 2}\right)-\frac{\left(V_{T G} K_{j 2}\right)^{2}}{4}
$$

in order to be used together with $T_{D}$ for the stability criteria statements.

\section{Closed-Loop System Lyapunov Function}

Using control law (5), the closed-loop system was rendered globally asymptotically stable, as was demonstrated in the analysis presented in [23]. Hence, the following Lyapunov function shall be considered throughout the paper:

$$
\begin{gathered}
V_{T}\left(q_{F}, \dot{q}_{F}\right)=\frac{1}{2} \dot{q}_{F}^{T} M(q) \dot{q}_{F}+\frac{1}{2} q_{E}^{T} T_{D} q_{E}+U_{g}(q)-U_{g}\left(q_{d}\right) \\
+q_{E}^{T}\left[\begin{array}{c}
\tau_{g}\left(q_{d}\right) \\
0
\end{array}\right] \geq 0
\end{gathered}
$$

, where $M(q)=\left[\begin{array}{cc}M_{J}(q) & 0 \\ 0 & J\end{array}\right], q_{F}=\left[\begin{array}{l}q \\ \theta\end{array}\right], q_{F_{d}}=\left[\begin{array}{l}q_{d} \\ \theta_{d}\end{array}\right], \quad q_{E}=$ $\left(q_{F}-q_{F_{d}}\right)$.

Explicit calculations proving that the time derivative is negative definite are omitted here as they can be found in [22]. It is essential to state that global stability holds if and only if the following conditions are satisfied [23]:

$$
\lambda_{\min }\left(T_{D}\right)>\alpha \text { and } \lambda_{\min }(\gamma)>0
$$

where $\lambda_{\text {min }}$ denotes the minimum Eigenvalue.

The satisfaction of these conditions is contingent upon the tuning of the controller, which in this case is related to the selection of the $K_{m 1}, K_{m 2}$ and $K_{j 2}$ matrices.

Furthermore, global stability does not imply the humanoid's possession of a dynamically balanced state in the CoP sense, and for this reason, the subsequent section will focus on describing and proving how the CoP deviation can be bounded by a constant multiplied by the square root of the value of the Lyapunov function (10).

\section{BALANCE MONITORING}

\section{A. Dynamical CoP Expression for Compliant Bipeds}

This sub-section provides an expression for a compliant biped's CoP, revealing that this variable may not be controlled in a direct manner, as opposed to a stiff biped's $\mathrm{CoP}$. We need to express the CoP in terms of the system's dynamics and shall commence with the following equation:

$$
X_{C o P}=\frac{\tau_{a}}{F_{Z}}
$$

with $\tau_{a}$ and $F_{Z}$ representing the ankle torque and the vertical component of the GRF, that may also be expressed as:

$$
F_{Z}=m_{T}\left(\ddot{Z}_{C}+g\right)
$$

where $m_{T}$ is the robot's total mass and $\ddot{Z}_{C}$ is the CoM acceleration along the $\mathrm{z}$-axis, while the acceleration of gravity, $g=9.81 \frac{\mathrm{m}}{\mathrm{s}^{2}}$. The relationship presented in [6] may now be employed, although it needs to be appropriately modified for the treatment of compliant humanoids beforehand. Thus, (1) - (2) may be expressed as follows:

$$
M \ddot{q}_{F}=\tau-W
$$

where $\tau=\left[\begin{array}{c}-S_{m}^{T} P\left(S_{m} q-\theta\right) \\ V_{T G} V_{m}\end{array}\right], W=\left[\begin{array}{l}-\tau_{g}(q)+(N+C) \dot{q} \\ -P\left(S_{m} q-\theta\right)+D \dot{\theta}\end{array}\right]$. By substituting (12) into (11) we obtain:

$$
X_{C o P}=\frac{\tau_{a}}{m_{T}\left(\ddot{Z}_{C}+g\right)}
$$

$\tau_{a}$ is the first component of the vector $\tau=$ $\left[\begin{array}{c}-S_{m}^{T} P\left(S_{m} q-\theta\right) \\ V_{T G} V_{m}\end{array}\right]$, so:

$$
X_{C o P}=\frac{-G \cdot S_{m}^{T} P\left(S_{m} q-\theta\right)}{m_{T}\left(\ddot{Z}_{C}+g\right)}
$$

where $G$ is a selection matrix that selects the ankle torque. The above equation sheds light on the fact that a compliant robot's CoP may not be directly controlled, since the torque input $\tau_{a}=-G \cdot S_{m}^{T} P\left(S_{m} q-\theta\right)$ is not directly controllable.

\section{B. Trigger Energy and CoP}

The aim is to obtain an energy value corresponding to a state that has been assumed by the robot, from which it may not be able to recover its balance [19], and to set this as the trigger energy. Given the fact that our dynamical model and energy function are concerned solely with sagittal motions, this would imply the CoP reaching either one of the $\mathrm{x}$-axis limits of the support polygon.

Lateral motions are not considered since the relevant joints do not incorporate compliant elements and would hence require a different control law that would be accompanied with a distinct Lyapunov function. It is noted that the robot is to be operated around the point of maximal balance which would imply $X_{C O P}=0$, that in our case corresponds to $q_{F_{d}}=0$. The method works not only for this specific operating point of 'maximal balance' but also for arbitrary equilibrium points. Furthermore, the trigger energy can be computed using equation (36) that is presented in the next sub-section, while the Lyapunov function (10) is to be used for the measurement of the total energy during the experiments. Thus, the difference between the measured and 
trigger energy values will provide insight into the robot's state of stability at a given time, as described in [19].

To elaborate, the energy value tends to increase as the CoP moves from the center of the polygon towards its edges, although the two are not related in a linear manner.

Additionally, this approach can be used for trajectory generation entailing models that are more intricate than the linear inverted pendulum model (LIPM).

\section{LYAPUNOV ENERGY-COP EXPRESSION}

\section{A. Relationship Formulation}

For a bipedal robot whose closed-loop energy is described by (10), there exists a range of energy values for which the $\mathrm{CoP}$ can only remain within the support polygon, as will be demonstrated in the subsequent lines.

It shall be assumed that the value of $\ddot{Z}_{C}$ is small in comparison to that of $g$, as it is in practice, and has also been considered in several other works [5][6][13], such that:

$$
X_{C o P}=\frac{\tau_{a}}{m_{T} g}
$$

where $\tau_{a}=-G \cdot S_{m}^{T} P\left(S_{m} q-\theta\right)$.

Additionally, we define the CoP position and the ankle torque at equilibrium $\left(X_{C o P_{d}}\right.$ and $\left.\tau_{d}\right)$ :

$$
X_{C o P_{d}}=\frac{\tau_{d}}{m_{T} g}
$$

with $\tau_{d}=-G \cdot S_{m}^{T} P\left(S_{m} q_{d}-\theta_{d}\right)$.

Besides, we recall that $\tau_{a}=-G \cdot S_{m}^{T} P\left(S_{m} q-\theta\right)$, hence:

$$
\begin{gathered}
\tau_{a}-\tau_{d}=-G S_{m}^{T} P\left(S_{m} q-S_{m} q_{d}-\theta+\theta_{d}\right) \\
\left|\tau_{a}-\tau_{d}\right| \leq\left\|G S_{m}^{T} P\right\|\left\|S_{m} q-S_{m} q_{d}-\theta+\theta_{d}\right\|
\end{gathered}
$$

By the triangle inequality:

$$
\begin{gathered}
\left|\tau_{a}-\tau_{d}\right| \leq\left\|G S_{m}^{T} P\right\|\left(\left\|S_{m} q-S_{m} q_{d}\right\|+\left\|\theta-\theta_{d}\right\|\right) \\
\left|\tau_{a}-\tau_{d}\right| \leq\left\|G S_{m}^{T} P\right\|\left(\left\|S_{m}\right\|\left\|q-q_{d}\right\|+\left\|\theta-\theta_{d}\right\|\right)
\end{gathered}
$$

while squaring both sides yields:

$$
\left|\tau_{a}-\tau_{d}\right|^{2} \leq\left\|G S_{m}^{T} P\right\|^{2}\left(\left\|S_{m}\right\|\left\|q-q_{d}\right\|+\left\|\theta-\theta_{d}\right\|\right)^{2}
$$

Since:

$$
\begin{aligned}
2\left\|S_{m}\right\|\left\|q-q_{d}\right\|\left\|\theta-\theta_{d}\right\| & \\
& \leq\left\|q-q_{d}\right\|^{2}+\left\|S_{m}\right\|^{2}\left\|\theta-\theta_{d}\right\|^{2}
\end{aligned}
$$$$
\text { we have: }
$$

$$
\begin{gathered}
\left(\left\|S_{m}\right\|\left\|q-q_{d}\right\|+\left\|\theta-\theta_{d}\right\|\right)^{2} \\
\leq\left(\left\|S_{m}\right\|^{2}+1\right)\left(\left\|q-q_{d}\right\|^{2}+\left\|\theta-\theta_{d}\right\|^{2}\right)
\end{gathered}
$$

Thus:

$$
\begin{gathered}
\left|\tau_{a}-\tau_{d}\right|^{2} \leq\left\|G S_{m}^{T} P\right\|^{2}\left(\left\|S_{m}\right\|^{2}+1\right)\left(\left\|q-q_{d}\right\|^{2}\right. \\
\left.+\left\|\theta-\theta_{d}\right\|^{2}\right) \\
\left|\tau_{a}-\tau_{d}\right|^{2} \leq\left\|G S_{m}^{T} P\right\|^{2}\left(\left\|S_{m}\right\|^{2}+1\right)\left(\left\|q_{E}\right\|^{2}\right)
\end{gathered}
$$

Finally $\left\|S_{m}\right\|=\sqrt{n_{\max }}$, with $n_{\max }=\max _{1 \leq \mathrm{i} \leq \mathrm{j}}\left(\mathrm{n}_{\mathrm{i}}\right)$ :

$$
\left\|q_{E}\right\|^{2} \geq \frac{1}{n_{\max }+1}\left(\frac{\left|\tau_{a}-\tau_{d}\right|}{\left\|G \cdot S_{m}^{T} P\right\|}\right)^{2}
$$

Additionally, the following statement holds true:

$$
\left\|q_{E}^{T} T_{D} q_{E}\right\| \geq \lambda_{\min }\left(T_{D}\right)\left\|q_{E}\right\|^{2}
$$

hence leading to the expression:

$$
\left\|\frac{1}{2} q_{E}^{T} T_{D} q_{E}\right\| \geq \frac{1}{2\left(n_{\max }+1\right)} \lambda_{\min }\left(T_{D}\right)\left(\frac{\left|\tau_{a}-\tau_{d}\right|}{\left\|G \cdot S_{m}^{T} P\right\|}\right)^{2}
$$

Since the $U_{g}(q)-U_{g}\left(q_{d}\right)+q_{E}^{T}\left[\begin{array}{c}\tau_{g}\left(q_{d}\right) \\ 0\end{array}\right]$ terms have thus far been neglected in the analysis, it shall now be proven that these are smaller than $\frac{1}{2} q_{E}^{T} T_{D} q_{E}$. Alternatively, it could be assumed that $U_{g}(q)=$ constant $=m \cdot g \cdot Z_{C}$, resulting in an LIPM with a fixed CoM height, although such an assumption shall not be made here. Recalling the following property of the potential energy:

$$
\left\|\frac{\partial^{2} U_{g}(q)}{\partial q^{2}}\right\|<\alpha
$$

which implies the following inequality, as shown in [23] :

$$
\left\|U_{g}(q)-U_{g}\left(q_{d}\right)+q_{E}^{T}\left[\begin{array}{c}
\tau_{g}\left(q_{d}\right) \\
0
\end{array}\right]\right\| \leq \frac{1}{2} \alpha\left\|q-q_{d}\right\|^{2}
$$

leads to:

$$
\begin{aligned}
\left\|U_{g}(q)-U_{g}\left(q_{d}\right)+q_{E}^{T}\left[\begin{array}{c}
\tau_{g}\left(q_{d}\right) \\
0
\end{array}\right]\right\| & \leq \frac{1}{2} \alpha\left\|q_{E}\right\|^{2} \\
\therefore\left\|U_{g}(q)-U_{g}\left(q_{d}\right)+q_{E}^{T}\left[\begin{array}{c}
\tau_{g}\left(q_{d}\right) \\
0
\end{array}\right]\right\| & \leq \frac{\alpha\left\|q_{E}^{T} T_{D} q_{E}\right\|}{2 \lambda_{\min }\left(T_{D}\right)}
\end{aligned}
$$

For an arbitrary equilibrium point $q_{F_{d}}$, the potential energy function $V_{P}\left(q_{F}\right)$ is given as:

$$
V_{P}\left(q_{F}\right)=\frac{1}{2} q_{E}^{T} T_{D} q_{E}+U_{g}(q)-U_{g}\left(q_{d}\right)+q_{E}^{T}\left[\begin{array}{c}
\tau_{g}\left(q_{d}\right) \\
0
\end{array}\right]
$$

By then using (30), we obtain:

$$
V_{P} \geq\left(1-\frac{\alpha}{\lambda_{\min }\left(T_{D}\right)}\right) \frac{\left\|q_{E}^{T} T_{D} q_{E}\right\|}{2}
$$


It is noted that:

$$
\frac{\alpha}{\lambda_{\min }\left(T_{D}\right)}<1
$$

Using (27) and simplifying, we obtain:

$$
V_{P} \geq \frac{1}{2\left(n_{\max }+1\right)}\left(\lambda_{\min }\left(T_{D}\right)-\alpha\right)\left(\frac{\left|\tau_{a}-\tau_{d}\right|}{\left\|G \cdot S_{m}^{T} P\right\|}\right)^{2}
$$

By then considering the total energy, through the addition of the positive (unless $\dot{q}_{F}=0$ ) $\frac{1}{2} \dot{q}_{F}^{T} M(q) \dot{q}_{F}$ term to the potential energy function, it is inferred that:

$$
\begin{gathered}
V_{T} \geq V_{P} \\
\Rightarrow \quad V_{T} \geq \frac{1}{2\left(n_{\max }+1\right)} \delta\left(\frac{\left|X_{C o P}-X_{C o P_{d}}\right| \cdot m_{T} g}{\left\|G \cdot S_{m}^{T} P\right\|}\right)^{2}
\end{gathered}
$$

where

$$
\delta=\left(\lambda_{\min }\left(T_{D}\right)-\alpha\right)>0
$$

The following expression is therefore acquired:

$$
\left|X_{C o P}-X_{C_{O} P_{d}}\right| \leq \frac{\sqrt{2\left(n_{\max }+1\right)}\left\|G \cdot S_{m}^{T} P\right\|}{\sqrt{\delta} \cdot m_{T} g} \sqrt{V_{T}}
$$

A relationship between the total energy and the $X_{C O P}$ has thus been established, while a number of calculations allow one to conclude the soundness of the units.

This may be viewed as the first proof demonstrating that for a specific low-level joint controller, $\mathrm{CoP}$ positions can be bounded by values that are proportional to the square root of a Lyapunov function (the total closed-loop energy). This property leads to the existence of critical energy values, below which it is certain that the CoP position is to forever remain within the support region, provided the absence of future external perturbations (i.e. energy injections). The analysis can be extended by considering any equilibrium point since its validity is not restricted to the $q_{F_{d}}=0$ configuration. Equation (36) can be used to compute the trigger energy values based on the $X_{C O P}$ values corresponding to the support polygon's limits. This provides a systematic approach to obtaining Regions of Trust, as opposed to defining them arbitrarily [25]. Although we have only considered a double support model in this work, it is evident that the same formulae can be applied to the single support phase with minor modifications. Due to the Lyapunov function's inclusion of the kinetic energy terms of all the joints, this method's applicability is not restricted to ankle strategies. Furthermore, for every energy value corresponding to a balanced state in terms of the $\mathrm{CoP}$ value, the controller will also ensure that the robot is asymptotically stable at the joint level, and as a result, this will also imply that the CoP converges to the desired equilibrium position. It should be pointed out that there is absolutely no difficulty in computing the numerical values of the variables constituting the final expression (36), i.e. $\delta, E$, $\left\|G \cdot S_{m}^{T} P\right\|$, and $n_{\max }$.

\section{B. Stepping Algorithm}

Prior to presenting a succinct version of our algorithm, it is important to state that for a given push, there will be two distinct positive energy values pertaining to the CoP's position in either the frontmost or rearmost sections of the support polygon. If the robot were agnostic to the direction of the push, it would then utilize the smallest of these two positive values to trigger a step. Since however our robot is equipped with FT sensors at the feet, these are used to measure the CoP position and hence the robot's direction of motion at a given point in time. Nevertheless, there is still a need for the observance of the center of pressure velocity, $\dot{X}_{C o P}$, that could be crucial in certain situations, such as ones in which the robot is moving towards a point that is close to either of the CoP limits and is then perturbed in the direction opposite to that of its current motion. The energy function itself would be agnostic to the fact that the velocity due to the disturbance was in fact a stabilizing one (i.e. returning it back to $X_{C O P}=0$ ) and would require further directional information, that could be directly provided by the $\dot{X}_{C O P}$. Hence, a prerequisite for the selection of a trigger energy is the $X_{C O P}$ 's and $\dot{X}_{C O P}$ 's possession of equal signs. Having elucidated these aspects of our strategy, we may now summarize the energy computation and balance monitoring algorithm:

1. The $E_{F}$ and $E_{B}$ energies required to drive the CoP to the front and rear edges of the support polygon can be computed either by using theoretical upper bounds given by equation (36), or through experimental means.

2. The $E_{F}$ and $E_{B}$ values are both set as trigger energies whose selection is dependent upon the $\mathrm{x}$-axis $\mathrm{CoP}$ measurements read by the force/torque sensors and the corresponding $\mathrm{CoP}$ velocities.

3. The energy value is monitored in real-time using (10), hence serving as an indicator of the humanoid's state of balance.

The $E_{F}$ and $E_{B}$ values may differ significantly, in accordance with the dimensions of the support polygon, although in the majority of cases, including the one considered in this paper, the $E_{F}$ value will tend to be larger.

\section{BALANCE MONITORING AND STEPPING EXPERIMENTS}

\section{A. Balance Monitoring Experiments}

The propounded method was corroborated through experimental means that involved the lower body of the COMAN [26][27][28] being placed on the floor and subjected to a series of perturbations.

The first set of tests served the purpose of demonstrating the validity of (36) by moving the robot's waist to arbitrary locations while it was in a double support stance. The force/torque sensors mounted on the robot's foot soles enabled the acquirement of $\mathrm{x}$-axis force readings that indicated the direction from which the push had been applied 
and thus the energy limit to be utilized for signaling whether or not the robot was stable. Fig. 2 displays the pertinent results, wherein it is evident that the real $\mathrm{CoP}\left(X_{C_{\text {OP }} \text { real }}\right)$ is always confined within the limits defined by the value of the theoretical bound $\left(X_{C O P_{T h}}\right)$, as described by (36), thus allowing us to conclude that the estimate is a conservative one guaranteeing that the CoP will reside within a safe region of operation at all times. Additionally, an imperative aspect of these results is that both the energy and the CoP asymptotically converge towards the equilibrium point, as described by (36). Moreover, Figs. 4 and 5 portray the evolution of the energy and the CoP throughout the course of the frontal pushing experiments, while the orange lines on each plot denote the actual energy $\left(E_{F E}, E_{B E}\right.$ in Table I) and $\mathrm{CoP}$ limits, and the green line represents the theoretical energy limits $\left(E_{F T}, E_{B T}\right.$ in Table I).

During the tests, the robot lost its balance at the instances when the energy exceeded the Energy $y_{\text {real }}$ and $X_{\text {CoP }}$ real values, hence signifying that the limits were accurately calculated. Fig. 5 also displays the energy decrease that occurs once the robot loses balance, and this is attributed to the fact that the humanoid was held in place and manually moved back to the equilibrium position once the energy limit was exceeded, in order to prevent it from hitting the floor and damaging its structure.

A similar experiment entailing the application of rear-side disturbances also permitted the observation that the robot's balance was lost once the energy threshold was exceeded, as depicted in Figs. 6 and 7.

Fig. 8 was acquired during experiments that involved getting hold of the robot's pelvis and moving it rapidly, in a reciprocating manner, leading to the generation of relatively higher joint velocities as compared to those seen in the above figures. Consequently, the discrepancy between the overall and potential energies is perspicuous, thus highlighting the importance of the kinetic energy in dynamical cases, as is to be expected.

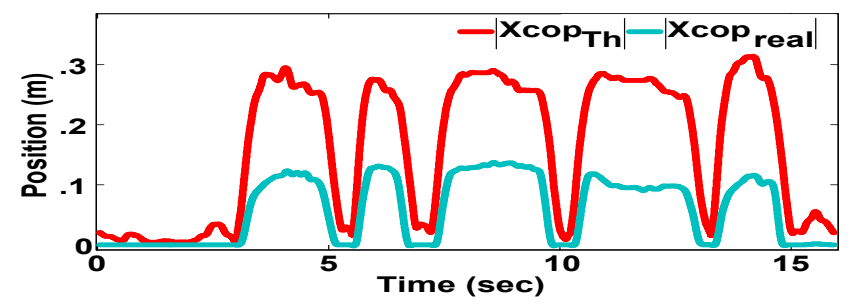

Figure 2. CoP response during handling test.

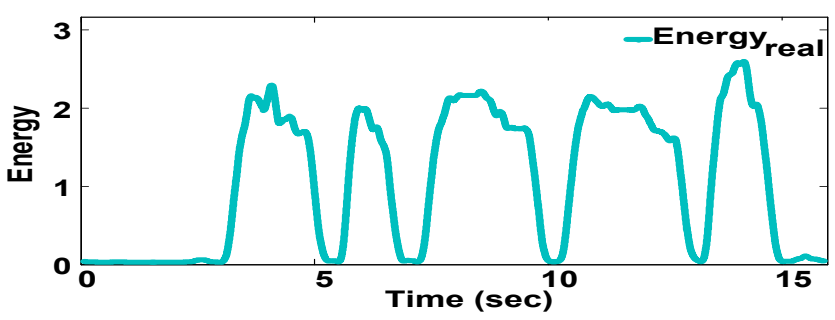

Figure 3. Energy response during handling test.
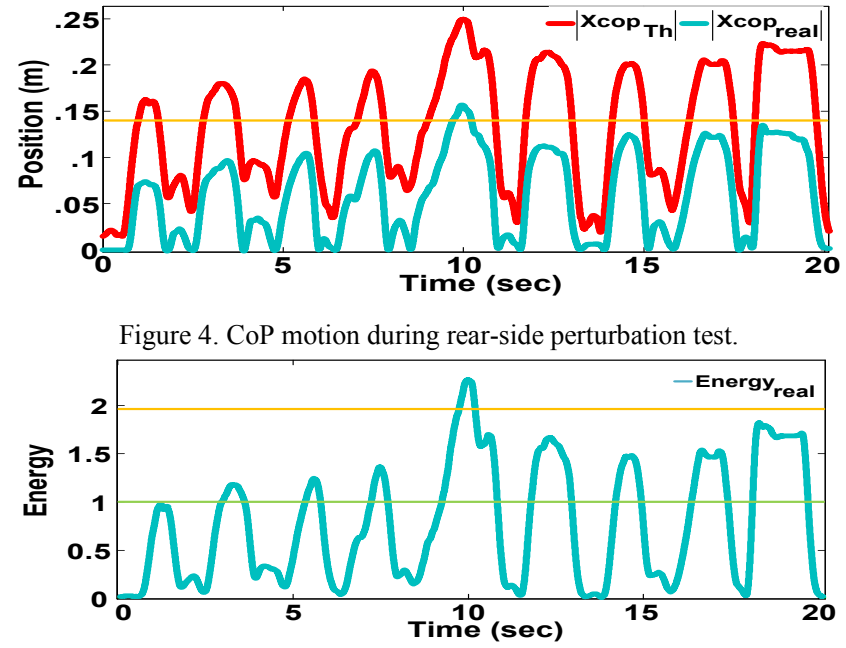

Figure 5. Energy responses during rear-side perturbation test.

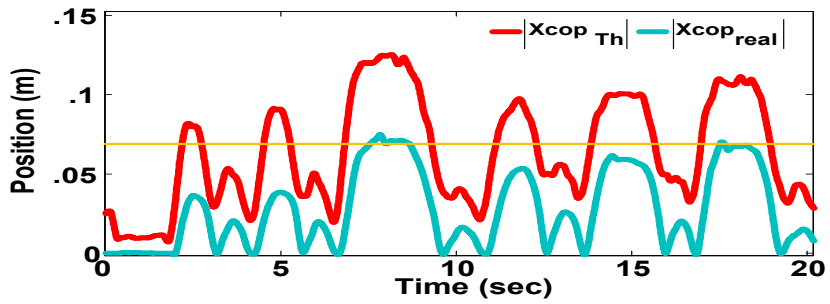

Figure 6. CoP motion during frontal perturbation test.

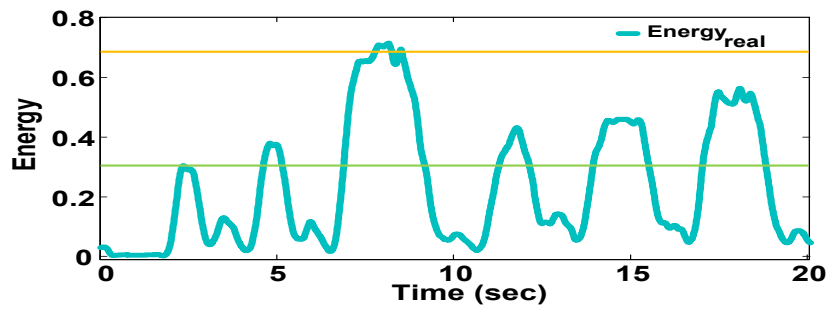

Figure 7. Energy response during frontal perturbation test.

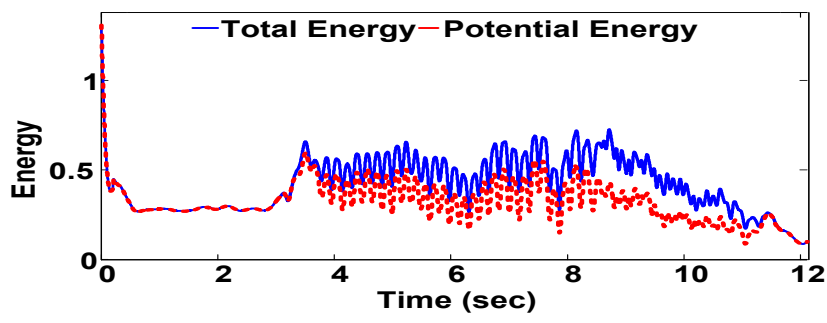

Figure 8. Energy response during robot's reciprocating motion.

TABLE I

TRIGGer ENERGY VALUES

\begin{tabular}{cc}
\hline Variable & Value \\
\hline$E_{F E}$ & 1.96 \\
$E_{F T}$ & 1.03 \\
$E_{B E}$ & 0.71 \\
$E_{B T}$ & 0.34 \\
\hline
\end{tabular}

\section{B. Stepping Recovery Experiments}

In order to demonstrate the practicability of the trigger energy values in terms of aiding in the balance maintenance 
of the system, a stepping recovery experiment was carried out, involving the COMAN being pushed while standing in an upright position and then using the trigger energy to perform a step, as shown in the video attachment.

\section{ACKNOWLEDGEMENT}

This work is supported by the WALK-MAN FP7-ICT-201310 European Commission project.

\section{CONCLUSIONS}

A balance monitoring strategy for humanoid robots was presented in the paper and experimentally validated on the COMAN. The main concept behind the proposed method was the use of a closed-loop energy function aimed at accurately describing bounds on the robot's state of stability at a given point in time. A mathematical proof pertaining to the validity of the proposed approach has been provided, by establishing a relationship between the CoP and the closedloop energy, thus also attesting the fact that for certain energy values, the CoP can only remain within the support polygon. This can be viewed as an extension of the Lyapunov Stability Margin concept that was initially propounded in [19]. Hence, our approach addresses the critical problem of robot balance in a mathematically sound way, and it proves that it is actually possible to compute Lyapunov Stability Margins that are not overly conservative, even when considering a complex control law that takes the passive elements of the robot actuation into account. In situations where the robot could perform various types of motion, tuning values becomes a feeble, impractical option. In such a context, the possibility of swiftly computing Lyapunov Stability Margins will enhance the degree of the robot's balance awareness, and its ability to make sound decisions based on the circumstances. Additionally, as safety becomes an increasingly important topic in robotics, the mathematical proofs associated with these Lyapunov Stability Margins will be invaluable in terms of certifying that a robot will always behave as expected.

\section{REFERENCES}

[1] M. Vukobratovic, B. Borovac, "Zero-moment point - Thirty Five Years of its Life," Int. J. of Humanoid Robotics, vol. 1, no. 1, pp. 157173, 2004

[2] P. Sardain, G. Bessonnet, "Forces Acting on a Biped Robot. Center of Pressure - Zero Moment Point," IEEE Trans. on Systems, Man, and Cybernetics, vol. 34, pp. 630-637, 2004.

[3] A. Goswami, "Postural Stability of Biped Robots and the FootRotation Indicator FRI Point," Int. J. of Robotics Research, vol. 18, pp. 523-533, 1999.

[4] B. Stephens, "Integral Control of Humanoid Balance," Proc. IEEE/RSJ Int. Conf. on Intelligent Robots and Systems, pp. 40204027, 2007.

[5] S. Kajita, K. Tani, "Study of Dynamic Biped Locomotion on Rugged Terrain," Proc. Int. Conf. on Advanced Robotics, vol. 1, pp. 741-746, 1991.

[6] B. Stephens, "Humanoid Push Recovery," Proc. IEEE-RAS Int. Conf. on Humanoid Robots, pp. 589-595, 2007.

[7] S. Hyon and G.Cheng, "Passivity-Based Full-Body Force Control for Humanoids and Application to Dynamic Balancing and Locomotion," Proc. IEEE/RSJ Int. Conf. on Intelligent Robots and Systems, pp. 4915-4922, 2006.
[8] Y. Kim, B. Lee, J. Ryu, J. Kim, "Landing Force Control for Humanoid Robot by Time-Domain Passivity Approach," IEEE Trans. on Robotics, vol. 23, no. 6, pp. 1294-1301, 2007.

[9] A. Goswami, V. Kallem, "Rate of change of angular momentum and balance maintenance of biped robots," Proc. IEEE Int. Conf. on Robotics and Automation, vol. 4, pp. 3785-3790, 2004.

[10] C. Azevedo, P. Poignet, B. Espiau, "Moving horizon control for biped robots without reference trajectory," Proc. IEEE Int. Conf. on Robotics and Automation, vol. 3, pp. 2762-2767, 2002.

[11] P. - B. Wieber, "Trajectory Free Linear Model Predictive Control for Stable Walking in the Presence of Strong Perturbations," Proc. IEEERAS Int. Conf. on Humanoid Robots, pp. 137 - 142, 2006.

[12] S. Kajita, F. Kanehiro, K. Kaneko, K. Fujiwara, K. Harada, K. Yokoi, H. Hirukawa, "Biped walking pattern generation by using preview control of zero-moment point," Proc. IEEE Int. Conf. on Robotics and Automation, vol. 2, pp. 1620-1626, 2003.

[13] Napoleon, S. Nakaura, M. Sampei, "Balance Control Analysis of Humanoid Robot based on ZMP Feedback Control," Proc. IEEE/RSJ Int. Conf. on Intelligent Robots and Systems, vol. 3, pp. 2437-2442, 2002.

[14] J. Pratt, J. Carff, S. Drakunov, A. Goswami, "Capture Point: A Step toward Humanoid Push Recovery," Proc. IEEE-RAS Int. Conf. on Humanoid Robots, pp. 200-207, 2006.

[15] R. Renner, S. Behnke, "Instability Detection and Fall Avoidance for a Humanoid Using Attitude Sensors and Reflexes," Proc. IEEE/RSJ Int Conf. on Intelligent Robots and Systems, pp. 2967-2973, 2006.

[16] P.-B. Wieber, "Viability and Predictive Control for Safe Locomotion," Proc. IEEE/RSJ Int. Conf. on Intelligent Robots and Systems, pp. 1103-1108, 2008.

[17] O. Höhn, W. Gerth, "Probabilistic Balance Monitoring for Bipedal Robots," Int. J. of Robotics Research, vol. 28, pp. 245-256, 2009.

[18] K. Yamamoto, "Maximal Output Admissible Set for Trajectory Tracking Control of Biped Robots and its Application to Falling Avoidance Control," Proc. IEEE/RSJ Int. Conf. on Intelligent Robots and Systems, pp. 3643-3648, 2013.

[19] P. -B. Wieber, "On the stability of walking systems," Proc. Int. Workshop on Human and Human Friendly Robotics, 2002.

[20] S. Kajita, T. Yamaura and A. Kobayashi, "Dynamic Walking Control of a Biped Robot Along a Potential Energy Conserving Orbit," IEEE Trans. on Robotics and Automation, vol. 8, no. 4, pp. 431-438, 1992.

[21] E. Garcia and P. G. de Santos, "An improved energy stability margin for walking machines subject to dynamic effects," Robotica, vol. 23, pp. 13-20, 2005 .

[22] E. Spyrakos-Papastavridis, G. Medrano-Cerda, N. G. Tsagarakis, J. S. Dai and D. G. Caldwell, "Gravity Compensation Control of Compliant Joint Systems with Multiple Drives," Proc. IEEE Int. Conf. on Robotics and Automation, pp.4960-4966, 2013.

[23] E. Spyrakos-Papastavridis, G. Medrano-Cerda, N. G. Tsagarakis, J. S Dai and D. G. Caldwell, "A Compliant Humanoid Walking Strategy Based on the Switching of State Feedback Gravity Compensation Controllers," Proc. IEEE/RSJ Int. Conf. on Intelligent Robots and Systems, pp. 3630-3636, 2013.

[24] C. Ott, A. Albu-Schäffer, A. Kugi, S. Stramigioli, G. Hirzinger, "A Passivity Based Cartesian Impedance Controller for Flexible Joint Robots - Part I: Torque Feedback and Gravity Compensation," Proc. IEEE Int. Conf. on Robotics and Automation, vol. 3, pp.2659-2665 2004.

[25] M. Giftthaler, K. Byl, "Increased robustness of humanoid standing balance in the sagittal plane through adaptive joint torque reduction," Proc. IEEE/RSJ Int. Conf. on Intelligent Robots and Systems, pp. 4130-4136, 2013.

[26] N. G. Tsagarakis, S. Morfey, G. A. Medrano-Cerda, Z. Li and D. G Caldwell, "Compliant Humanoid COMAN: Optimal Joint Stiffness Tuning for Modal Frequency Control," Proc. IEEE Int. Conf. on Robotics and Automation, pp. 673-678, 2013

[27] N.G.Tsagarakis, Matteo Laffranchi, Bram Vanderborght and Darwin G. Caldwell, "A Compact Soft Actuator Unit for Small Scale Human Friendly Robots," Proc. IEEE Int. Conf. on Robotics and Automation, pp. 4356-4362, 2009.

[28] N.G. Tsagarakis, Zhibin Li, Jody Alessandro Saglia, Darwin G. Caldwell, "The Design of the Lower Body of the Compliant Humanoid Robot "cCub"", Proc. IEEE Int. Conf. on Robotics and Automation, pp. 2035 - 2040, 2011. 